\title{
Comorbidity in Adult Bone Sarcoma Patients: A Population-Based Cohort Study
}

\author{
Ninna Aggerholm-Pedersen, ${ }^{1,2,3}$ Katja Maretty-Nielsen, ${ }^{1,2}$ Johnny Keller, ${ }^{1,4}$ \\ Steen Baerentzen, ${ }^{1,5}$ and Akmal Safwat ${ }^{1,3}$ \\ ${ }^{1}$ Sarcoma Centre of Aarhus University Hospital, Norrebrogade 44, 8000 Aarhus, Denmark \\ ${ }^{2}$ Department of Experimental Clinical Oncology, Aarhus University Hospital, Norrebrogade 44, Building 5, 8000 Aarhus, Denmark \\ ${ }^{3}$ Department of Oncology, Aarhus University Hospital, Norrebrogade 44, Building 5, 8000 Aarhus, Denmark \\ ${ }^{4}$ Department of Orthopedic Surgery E5, Aarhus University Hospital, Norrebrogade 44, Building 7, 8000 Aarhus, Denmark \\ ${ }^{5}$ Department of Pathology, Aarhus University Hospital, Norrebrogade 44, Building 18, 8000 Aarhus, Denmark
}

Correspondence should be addressed to Ninna Aggerholm-Pedersen; aggerholm@oncology.dk

Received 3 December 2013; Revised 28 January 2014; Accepted 28 January 2014; Published 27 February 2014

Academic Editor: Clement Trovik

Copyright (C) 2014 Ninna Aggerholm-Pedersen et al. This is an open access article distributed under the Creative Commons Attribution License, which permits unrestricted use, distribution, and reproduction in any medium, provided the original work is properly cited.

\begin{abstract}
Background. Comorbidity is an important prognostic factor for survival in different cancers; however, neither the prevalence nor the impact of comorbidity has been investigated in bone sarcoma. Methods. All adult bone sarcoma patients from western Denmark treated at the Aarhus Sarcoma Centre in the period from 1979 to 2008 were identified through a validated populationbased database. Charlson Comorbidity Index scores were computed, using discharge diagnoses from the Danish National Patient Registry. Survival was assessed as overall and disease-specific mortality. The impact of comorbidity was examined as rates according to the level of comorbidity as well as uni- and multivariately using proportional hazard models. Results. A total of 453 patients were identified. The overall prevalence of comorbidity was $19 \%$. The prevalence increased with age and over the study period. In patients with Ewing/osteosarcoma, comorbidity was not associated with an increased overall or disease-specific mortality. However, patients with bone sarcomas other than Ewing/osteosarcoma had increased overall mortality. Independent prognostic factors for diseasespecific survival were age, tumor size, stage at diagnosis, soft tissue involvement, grade, and surgery. Conclusion. The prevalence of comorbidity in bone sarcoma patients is low. Comorbidity impaired survival in patients with non-Ewing/nonosteosarcoma, histology. This emphasizes the importance of not only treating the sarcoma but also comorbidity.
\end{abstract}

\section{Introduction}

Bone sarcoma is a rare disease, with an incidence of approximately 8 cases per million/year. It occurs in all ages but has a characteristic bimodal distribution, with peak incidences for adolescents and elderly [1]. Changes in the general population are expected in the future, resulting in an increased population of elderly. The treatment of these is often complicated by the presence of chronic diseases, for example, comorbidity, which may impact survival. Comorbidity is an important prognostic factor for survival in other cancers, such as head and neck, renal, and bladder cancer [2-7]. Several factors have previously been identified as prognostic in bone sarcoma patients; however the impact of comorbidity has not been investigated previously.

The structure of the Danish health care system, with free of charge health care for all residents, and the extensive use of population-based health registries provide a unique possibility to examine the impact of comorbidity in large population-based series. We have just published an article covering the impact of comorbidity on overall survival in soft tissue sarcoma patients treated in the same institute over the same period of time. Because of the differences in age distribution, prognosis, pathological types, treatment modalities, and outcome between adult soft tissue and bone sarcomas, we chose to report the results in two separate 


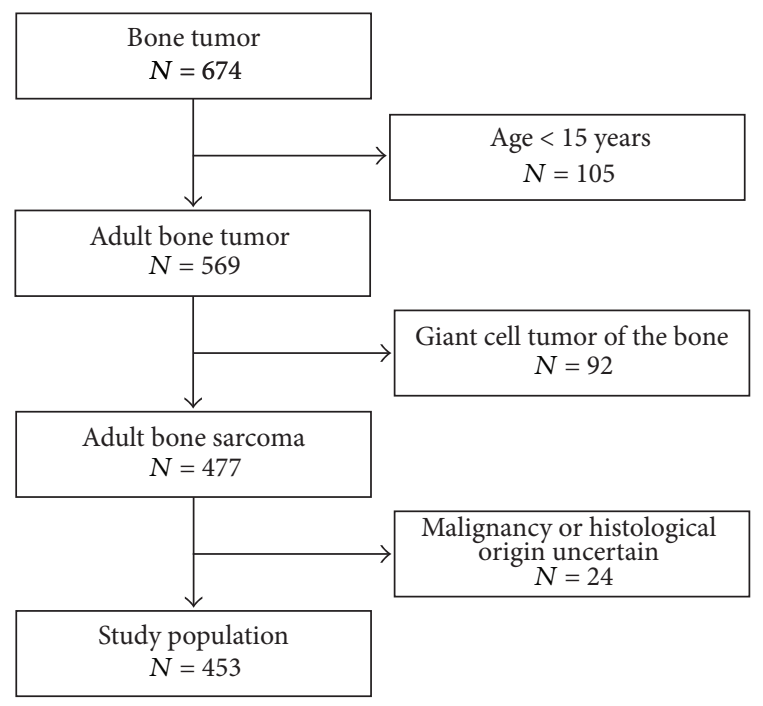

FIGURE 1: Flow chart for patients diagnosed with a bone sarcoma at the Sarcoma Centre of Aarhus University Hospital in the period from 1979 to 2008 .

publications. The aim of this study was therefore to examine the prevalence of comorbidity and its impact on survival among adult bone sarcoma patients.

\section{Patients and Methods}

The population of western Denmark is approximately 2.5 million [8]. All Danish residents are assigned a unique 10-digit civil personal registration number (CPR number), rendering individual linkage throughout all Danish registries possible $[9,10]$.

2.1. Identification of Bone Sarcoma Patients. Since 1979 all sarcoma patients treated at the Aarhus Sarcoma Centre have been registered in a population-based clinical database, the Aarhus Sarcoma Registry [1]. Patients at the Aarhus Sarcoma Centre are diagnosed and treated, according to national guidelines, by an experienced multidisciplinary sarcoma team $[11,12]$. Bone sarcomas were classified primarily as low or high grade, with the exception of chondrosarcoma being classified as low, intermediate, or high grade.

Between 1979 and 2008, 651 consecutive patients were treated for bone sarcomas or related lesions at the Sarcoma Centre of Aarhus University Hospital, Denmark. Patients were excluded as shown in Figure 1. The study population comprised 453 adult patients.

2.2. Comorbidity. The National Patient Registry (NPR) contains information on all somatic patients admitted to Danish hospitals since 1977, as well as outpatient visits since 1995 [1316]. Registered data includes CPR number, admission and discharge dates, as well as all discharge diagnoses according to the eighth (prior to 1994) or tenth version of the International Classification of Disease (ICD-8 and ICD-10). The registry covers more than $99 \%$ of all Danish hospital admissions [16].
TABLE 1: Prevalence and scores of medical conditions as listed in the Charlson Comorbidity Index among adult bone sarcoma patients treated at the Aarhus Sarcoma Centre between 1979 and 2008 ( $N=$ 453).

\begin{tabular}{|c|c|c|c|}
\hline Conditions & $N$ & $\%$ & Score \\
\hline Myocardial infarct & 9 & 2.0 & 1 \\
\hline Congestive heart failure & 5 & 1.1 & 1 \\
\hline Peripheral vascular disease & 12 & 2.7 & 1 \\
\hline Cerebrovascular disease & 9 & 2.0 & 1 \\
\hline Dementia & 0 & 0.0 & 1 \\
\hline Chronic pulmonary disease & 15 & 3.3 & 1 \\
\hline Connective tissue disease & 6 & 1.3 & 1 \\
\hline Ulcer disease & 10 & 2.2 & 1 \\
\hline Mild liver disease & 1 & 0.2 & 1 \\
\hline Diabetes & 11 & 2.4 & 1 \\
\hline Hemiplegia & 0 & 0.0 & 2 \\
\hline Moderate/severe renal disease & 2 & 0.4 & 2 \\
\hline Diabetes with end organ damage & 1 & 0.2 & 2 \\
\hline Any tumor ${ }^{\mathrm{a}}$ & 25 & 5.5 & 2 \\
\hline Leukemia & 3 & 0.7 & 2 \\
\hline Lymphoma & 3 & 0.7 & 2 \\
\hline Moderate/severe liver disease & 0 & 0.0 & 3 \\
\hline Metastatic solid tumor & 8 & 1.8 & 6 \\
\hline AIDS & 0 & 0.0 & 6 \\
\hline
\end{tabular}

${ }^{a}$ Excluding tumors in soft tissue and bone (ICD-8; 170, 171, 192.49-99 and ICD-10; C40-C41, C47, C49).

Comorbidity was assessed using the Charlson Comorbidity Index [17]. The Charlson Comorbidity Index was originally developed to predict 1-year mortality in a cohort of 559 medical patients and has later been adapted for usage with ICD-based hospital discharge data [18]. The index includes 19 medical conditions, which are weighted from 1 to 6 (Table 1) according to the risk of mortality and added to form a final score [17]. The included ICD codes are shown in Supplementary Materials (see Supplementary Table 1 in Supplementary Materials available online at http://dx.doi.org/10.1155/2014/690316).

The 453 adult bone sarcoma patients in the ASR were linked through their CPR number to the NPR and all discharge diagnoses registered before the date of the sarcoma diagnosis were extracted. Based on these diagnoses, a Charlson Comorbidity score for each patient was computed. All discharge diagnoses of 30 days and all cancer diagnoses of 90 days prior to the sarcoma diagnosis were excluded to eliminate diagnoses related to the sarcoma.

2.3. Survival. Survival was assessed using overall and diseasespecific mortality as endpoints. Patients were followed from the date of sarcoma diagnosis until death, emigration, or end of the study period. Data on death was obtained through the Danish Civil Registration system, which was established in 1968 and comprises current and historical information on Danish residents. Data includes CPR number, date of birth, 
TABLE 2: Uni- and multivariate analyses of comorbidity and possible important prognostic factors for overall and disease-specific mortality in adult bone sarcoma patients $(N=453)$.

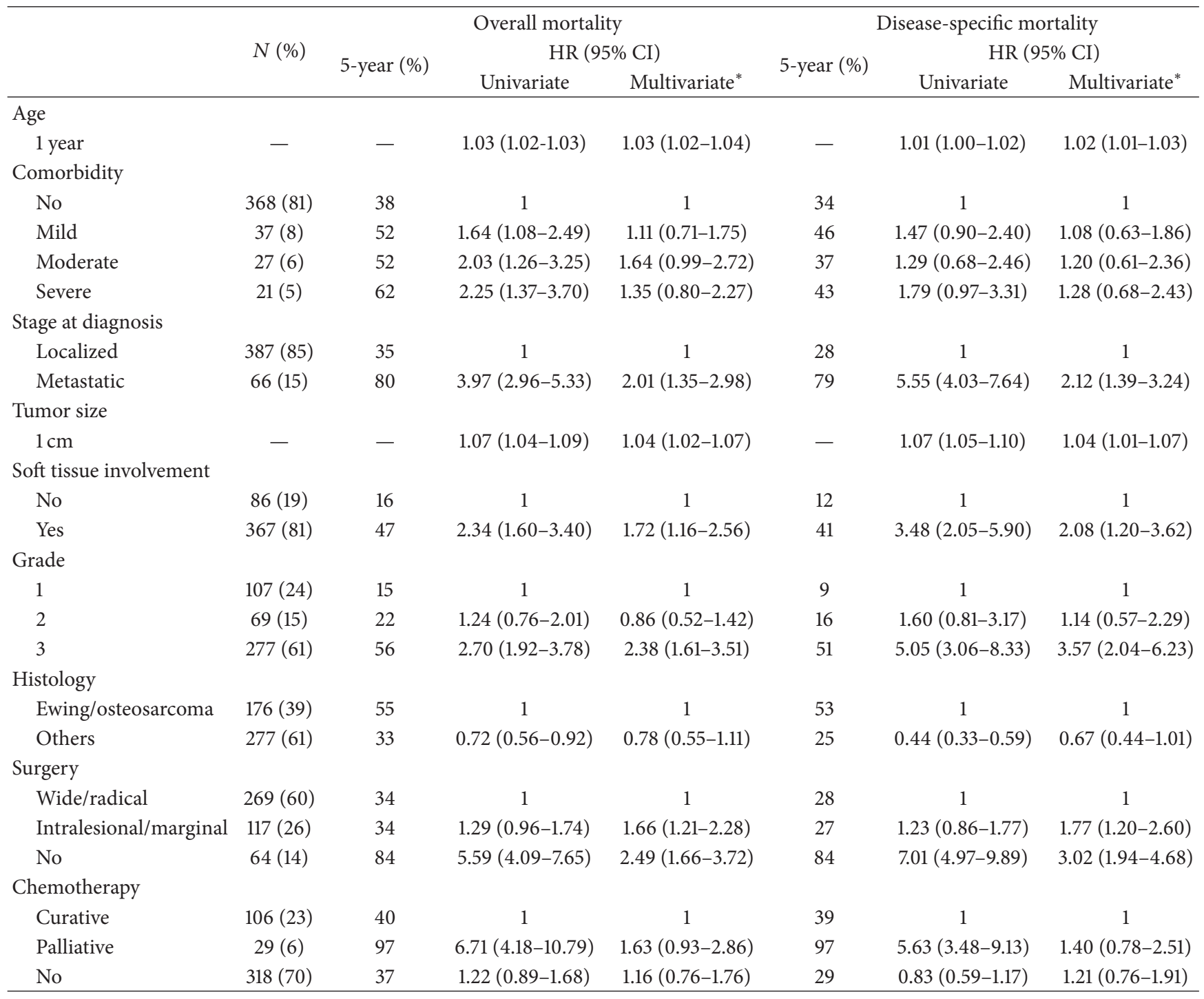

Note: abbreviations: HR: hazard ratio, CI: confidence interval. ${ }^{*}$ Multivariate analyses adjusted mutually for age, comorbidity, stage at diagnosis, tumor size, soft tissue involvement, grade, histology type, surgery, and chemotherapy.

residence, vital status (dead/alive), and date of death. The vital status is updated on a daily basis $[9,10]$.

The cause of death was obtained from the ASR and the Danish Cause of Death Registry [19]. The Danish Cause of Death Registry was initiated in 1875 based on the mandatory completion of death certificates for any death occurring in Denmark. The registry contains medical information from the death certificates including the immediate and underlying cause of death according to the ICD-8 and ICD-10 [19]. Disease-specific mortality was defined as death from sarcoma (ICD-8; 170, 171, 192.49-99 and ICD-10; C40-C41, C47, C49) or death with known metastatic disease.

2.4. Statistical Analyses. Comorbidity was analyzed as a categorical value based on the Charlson Comorbidity score as follows: no (score 0 ), mild (score 1), moderate (score 2), and severe (score $\geq 3$ ) comorbidities.The prevalence and type of comorbidity were assessed as proportions. Patient characteristics according to the level of comorbidity were analyzed using the Mann-Whitney $U$ test and the chi-square test. Overall and disease-specific mortality was assessed as rates with $95 \%$ confidence intervals (CI) and presented as cumulative incidence functions according to the level of comorbidity [20-23]. The association between comorbidity and mortality was assessed both uni- and multivariately, adjusting for the following possible prognostic factors: age, stage at diagnosis, tumor size, soft tissue involvement, grade, histological type, surgery, and chemotherapy. These were included as seen in Table 2. The correlation between the continuous variables (age and tumor size) and mortality was examined using the likelihood ratio test, comparing models with inclusion of the variables as continuous linear 
TABle 3: Patient characteristics by Charlson Comorbidity Index score $(N=453)$.

\begin{tabular}{|c|c|c|c|c|c|c|}
\hline & \multirow{2}{*}{$N(\%)$} & \multicolumn{4}{|c|}{ Charlson Comorbidity Index score $N(\%)$} & \multirow{2}{*}{$P$-value } \\
\hline & & 0 & 1 & 2 & $3+$ & \\
\hline Number & $453(100)$ & $368(81)$ & $37(8)$ & $27(6)$ & $21(5)$ & \\
\hline \multicolumn{7}{|l|}{ Age (years) } \\
\hline Median (range) & $46(15-90)$ & $40(15-90)$ & $62(17-83)$ & $59(17-81)$ & $64(21-86)$ & $<0.001$ \\
\hline \multicolumn{7}{|l|}{ Sex } \\
\hline Female & $179(40)$ & $137(37)$ & $20(54)$ & $12(44)$ & $10(48)$ & \\
\hline Male & $274(60)$ & $231(63)$ & $17(46)$ & $15(56)$ & $11(52)$ & 0.18 \\
\hline \multicolumn{7}{|l|}{ Year of diagnosis } \\
\hline 1979-1988 & $131(29)$ & $116(32)$ & $10(27)$ & $0(0)$ & $5(24)$ & \\
\hline 1989-1998 & $129(28)$ & $103(28)$ & $8(22)$ & $12(44)$ & $6(29)$ & \\
\hline 1999-2008 & $193(43)$ & $149(40)$ & $19(51)$ & $15(56)$ & $10(48)$ & 0.027 \\
\hline \multicolumn{7}{|l|}{ Stage at diagnosis } \\
\hline Localized & $387(85)$ & $321(87)$ & $32(86)$ & $18(67)$ & $16(76)$ & \\
\hline Metastatic & $66(15)$ & $47(13)$ & $5(14)$ & $9(33)$ & $5(24)$ & 0.018 \\
\hline \multicolumn{7}{|l|}{ Tumor size $(\mathrm{cm})$} \\
\hline Median (range) ${ }^{\mathrm{a}}$ & $8(1-30)$ & $8(1-30)$ & $8(2-30)$ & $7(2-15)$ & $10(3-23)$ & 0.14 \\
\hline \multicolumn{7}{|l|}{ Soft tissue involvement } \\
\hline No & $86(19)$ & $71(19)$ & $5(14)$ & $4(15)$ & $6(29)$ & \\
\hline Yes & $367(81)$ & $297(81)$ & $32(86)$ & $23(85)$ & $15(71)$ & 0.51 \\
\hline \multicolumn{7}{|l|}{ Malignancy grade } \\
\hline 1 & $107(24)$ & $86(23)$ & $11(30)$ & $7(26)$ & $3(14)$ & \\
\hline 2 & $69(15)$ & $55(15)$ & $8(22)$ & $4(15)$ & $2(10)$ & \\
\hline 3 & $277(61)$ & $227(62)$ & $18(50)$ & $16(59)$ & $16(76)$ & 0.59 \\
\hline \multicolumn{7}{|l|}{ Histological type } \\
\hline Ewing/osteosarcoma & $176(39)$ & $149(40)$ & $10(27)$ & $9(33)$ & $8(38)$ & \\
\hline Others & $277(61)$ & $219(60)$ & $27(73)$ & $18(67)$ & $13(62)$ & 0.40 \\
\hline \multicolumn{7}{|l|}{ Treatment } \\
\hline Surgery & $389(86)$ & $325(88)$ & $30(81)$ & $19(70)$ & $15(71)$ & 0.009 \\
\hline \multicolumn{7}{|l|}{ Type $^{b}$} \\
\hline Resection & $257(66)$ & $213(66)$ & $19(63)$ & $13(76)$ & $12(80)$ & \\
\hline Amputation & $130(34)$ & $112(34)$ & $11(37)$ & $4(24)$ & $3(20)$ & $<0.001$ \\
\hline \multicolumn{7}{|l|}{ Margin $^{c}$} \\
\hline Wide/radical & $269(70)$ & $228(70)$ & $19(63)$ & $13(76)$ & $9(60)$ & \\
\hline Intralesional/marginal & $117(30)$ & $96(30)$ & $11(37)$ & $4(24)$ & $6(40)$ & $<0.001$ \\
\hline Radiotherapy & $83(18)$ & $71(19)$ & $5(14)$ & $3(11)$ & $4(19)$ & 0.63 \\
\hline Chemotherapy & $135(30)$ & $119(32)$ & $4(11)$ & $8(30)$ & $4(19)$ & 0.005 \\
\hline Curative & $106(79)$ & $98(82)$ & $2(50)$ & $3(38)$ & $3(75)$ & \\
\hline Palliative & $29(21)$ & $21(18)$ & $2(50)$ & $5(63)$ & $1(25)$ & 0.012 \\
\hline
\end{tabular}

Note: ${ }^{a}$ tumor size: 38 missing. ${ }^{b}$ Type of surgery: 2 missing. ${ }^{c}$ Surgical margin: 3 missing.

and as four-knotted cubic splines, respectively. No significant difference between the respective models was found (overall mortality: age $P=0.11$, tumor size $P=0.22$; disease specific mortality: age $P=0.73$, tumor size $P=0.13$ ), and age and tumor size were thus included as continuously linear variables [24-26]. Missing data on tumor size and margin were computed using multiple imputations by chained equations [27]. Crude and adjusted hazard ratios with 95\% CI were computed using the Cox proportional hazard model. The proportional hazard assumption was assessed graphically. Disease-specific mortality was analyzed with death from other causes as a competing risk [28]. Effect modification was tested using the likelihood ratio test and assessed according to the principles described by Oxman and Guyatt [29]. A significant interaction between comorbidity and the histological subtype was encountered $(P=0.0003)$ and stratum-specific hazard ratios were thus computed. All tests were two-sided and a $P$ value $\leq 0.05$ was considered significant. Analyses were employed using Stata, version 13.0.

2.5. Ethics. This study was approved by the Danish Data Protection Agency and the Danish Health and Medicines Authority. 
TABLE 4: Histological subtypes in adult bone sarcoma patients treated at the Sarcoma Centre of Aarhus University Hospital from 1979 to $2008(N=453)$.

\begin{tabular}{lcc}
\hline Subtype & $N$ & $(\%)$ \\
\hline Chondrosarcoma & 197 & 43.5 \\
Osteosarcoma & 136 & 30.0 \\
Ewing sarcoma & 40 & 8.8 \\
Malignant fibrous histiocytoma & 33 & 7.3 \\
Chordoma & 21 & 4.6 \\
Malignant giant cell tumor & 9 & 2.0 \\
Angiosarcoma & 7 & 1.6 \\
Leiomyosarcoma & 5 & 1.1 \\
Others & 3 & 0.7 \\
Unclassifiable & 2 & 0.4 \\
\hline
\end{tabular}

\section{Results}

3.1. Patient Characteristics. Overall, 453 adult patients were diagnosed with a bone sarcoma in western Denmark between 1979 and 2008. The median age was 46 years (range 15-90) and $60 \%$ were males. The patient characteristics according to the Charlson Comorbidity Index score are shown in Table 3. The level of comorbidity was significantly associated with increased age, diagnosis in the last part of the study period, no surgery, a higher proportion of amputations, intralesional/marginal excisions, chemotherapy, and palliative treatment. As seen in Table 4, the most frequent histological types were chondrosarcoma and osteosarcoma. The median followup was 5.9 years (range $0.0-34.1$ ).

3.2. Prevalence of Comorbidity. Comorbidity was present in 85 of the 453 adult bone sarcoma patients, corresponding to a prevalence of $19 \%$. The prevalence of comorbidity was $15 \%$ and $21 \%$ for patients with Ewing/osteosarcoma and nonEwing/nonosteosarcoma, respectively. Mild comorbidity was seen in $44 \%$ of the patients with comorbidity, while moderate and severe comorbidities were seen in $32 \%$ and $25 \%$, respectively. As seen in Table 1, the most frequent type of comorbidity was "any tumor," which was seen in $5.5 \%$ of the patients. The prevalence of overall comorbidity increased with increasing age, being most frequent at 86 years where $44 \%$ of the patients had comorbidity (Figure $2(a)$ ). As seen in Figure 2(b) the prevalence of comorbidity increased over the study period, from $6 \%$ in 1979 to $26 \%$ in 2008 . The prevalence of severe comorbidity was nearly constant, while mild and moderate comorbidities increased over time from $1 \%$ to $10 \%$ and from $0 \%$ to $12 \%$, respectively.

3.3. Overall Mortality. In total, the 5- and 10 -year overall mortality was $41 \%$ (95\% CI 37-46) and 52\% (95\% CI $47-$ 57), respectively. The crude overall mortality was significantly higher in patients with comorbidity, independent of the level, as shown in Figure 3(a) and Table 2. Increasing age and tumor size, metastases at diagnosis, soft tissue involvement, high grade, and intralesional/marginal excision or no surgery was independently significantly associated with an increased overall mortality (Table 2). Moderate and severe comorbidities were found to be independent, significant prognostic factors for overall mortality in patients with nonEwing/nonosteosarcoma histology. Comorbidity was not associated with increased mortality in Ewing/osteosarcoma patients (Table 5).

3.4. Disease-Specific Mortality. In total 188 of the 453 patients died of their bone sarcoma or with metastatic disease, corresponding to a 5- and 10-year disease-specific mortality of $36 \%$ (95\% CI 31-40) and 40\% (95\% CI 36-45), respectively. The cumulative incidence function of the crude disease-specific mortality by level of comorbidity is shown in Figure 3(b). For patients without comorbidity, the crude 5-year diseasespecific mortality was 34\% (95\% CI 30-39), while for patients with mild, moderate, and severe comorbidities it was $46 \%$ (95\% CI 30-62), 37\% (95\% CI 19-55), and $43 \%$ (95\% CI 22-64), respectively. The level of comorbidity was not significantly correlated with disease-specific mortality in neither the uni- nor multivariate analysis as shown in Tables 2 and 5. Independent adverse prognostic factors were increasing age and tumor size, metastasis at diagnosis, soft tissue involvement, high grade, and intralesional/marginal excision or no surgery.

\section{Discussion}

In this population-based study of 453 adult bone sarcoma patients we found an overall prevalence of comorbidity of $19 \%$. The prevalence of comorbidity increased with an increasing age and over the study period. Independent adverse prognostic factors for disease-specific survival were increasing age and tumor size, metastasis at diagnosis, soft tissue involvement, high grade, and intralesional/marginal excision or no surgery. Moderate and severe comorbidities were significantly associated with overall survival in patients with non-Ewing/nonosteosarcoma histology, even when adjusting for important prognostic factors.

4.1. Methodological Reflections. The main strength of our study is the large sample size as well as population-based data with complete followup on all patients, facilitated by the structure of the Danish health care system. The information on comorbidity was extracted from an administrative registry. The potential information bias is considered low, especially compared to studies based on self-administered questionnaires or medical files, where comorbidity is suspected to be underreported. Coding errors in an administrative registry are expected to some extent; however since the comorbidity occurred before the sarcoma diagnosis, any misclassification is expected to be nondifferential [30]. One of the limitations of this study is the fact that outpatient data was only registered after 1995, indicating that minor comorbidity not requiring hospital admission is only captured in the last half of the study period. Furthermore the NPR was initiated in 1977 , leaving only two years of registered information on comorbidity for patients diagnosed with bone sarcoma in 1979. Yet, the increase in prevalence was uniform throughout 
TABLE 5: Multivariate analyses for the effect of comorbidity on overall and disease-specific mortality according to histological subtype.

\begin{tabular}{|c|c|c|c|c|c|}
\hline \multirow{2}{*}{ Histological subtype } & \multirow{2}{*}{ No. of patients } & \multicolumn{2}{|c|}{ Overall mortality } & \multicolumn{2}{|c|}{ Disease-specific mortality } \\
\hline & & 5 -year $(\%)$ & HR (95\% CI) & 5-year (\%) & HR (95\% CI) \\
\hline \multicolumn{6}{|l|}{ Ewing/osteosarcoma } \\
\hline No & 149 & 54 & 1 & 53 & 1 \\
\hline Mild & 10 & 70 & $1.02(0.43-2.42)$ & 60 & $0.80(0.31-2.09)$ \\
\hline Moderate & 9 & 67 & $1.12(0.47-2.66)$ & 56 & $0.88(0.33-2.36)$ \\
\hline Severe & 8 & 38 & $0.79(0.27-2.32)$ & 37 & $0.67(0.20-2.26)$ \\
\hline \multicolumn{6}{|l|}{ Others } \\
\hline No & 219 & 30 & 1 & 22 & 1 \\
\hline Mild & 27 & 45 & $1.42(0.81-2.48)$ & 41 & $1.54(0.78-3.05)$ \\
\hline Moderate & 18 & 44 & $2.36(1.18-4.70)$ & 28 & $1.05(0.37-2.97)$ \\
\hline Severe & 13 & 77 & $2.44(1.30-4.58)$ & 46 & $2.07(0.94-4.56)$ \\
\hline
\end{tabular}

Note: abbreviations: HR: hazard ratio, CI: confidence interval. Adjusted for age, stage at diagnosis, tumor size, soft tissue involvement, malignancy grade, surgical margin, and chemotherapy.

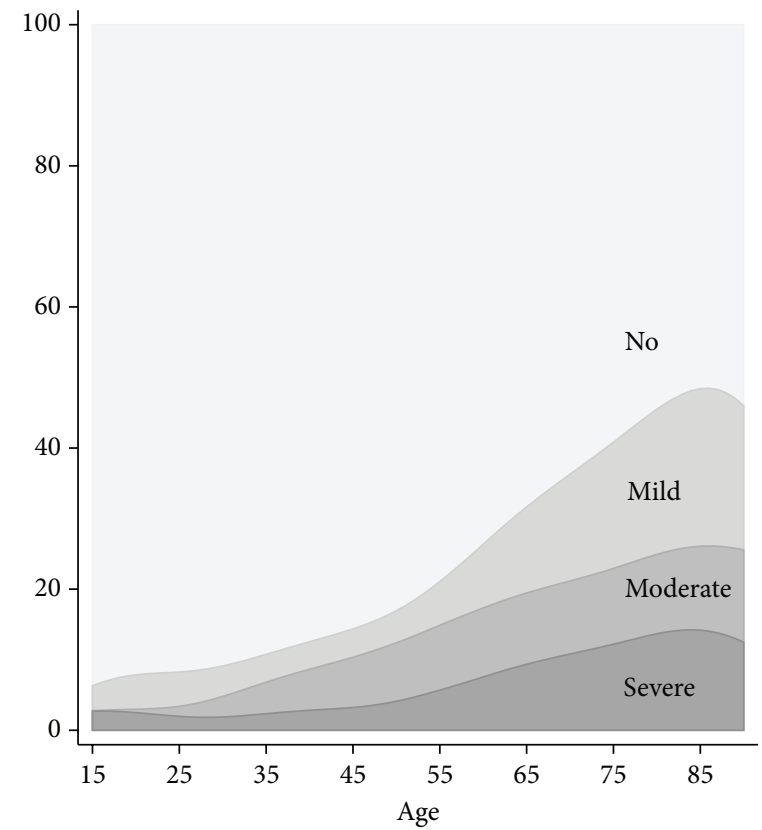

(a)

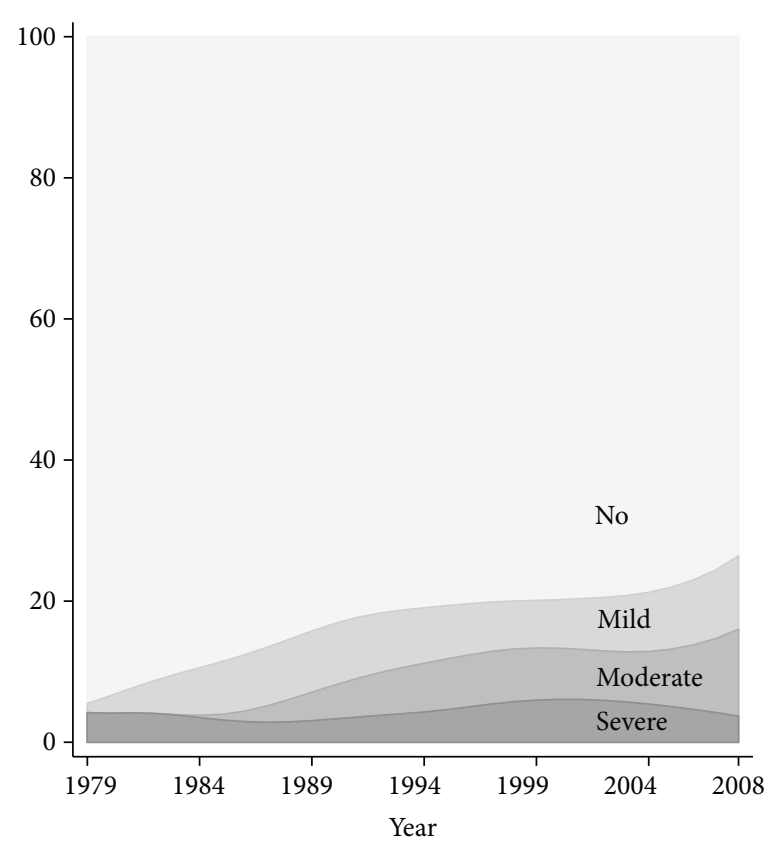

(b)

FIGURE 2: The prevalence of comorbidity as a percentage by age (a) and calendar year of diagnosis (b) in adult bone sarcoma patients treated at the Sarcoma Centre of Aarhus University Hospital in the period from 1979 to $2008(N=453)$.

the entire study period suggesting that comorbidity missed on this basis is minor.

The use of the ICD-10 codes in the NPR for the medical conditions in the Charlson Comorbidity Index has previously been validated. Thygesen et al. [31] reported an overall positive predictive value of $98 \%$ for the 19 conditions. The lowest positive predictive value was seen for diabetes mellitus with end organ damage $(82 \%)$. The prevalence of some of the milder conditions is expected to be underestimated in the NPR, since low-prevalent, severe diseases generally tend to have high negative predictive value, while high-prevalent, mild diseases tend to have lower negative predictive values. However the negative predictive value for ICD coding in the NPR has, to our knowledge, not been investigated.
The Charlson Comorbidity Index has previously been validated for various cancer types [3-6, 18, 32, 33]. The index does not perfectly adjust for comorbidity since some of the medical conditions with the same weight have different outcomes, for example, myocardial infarction and connective tissue disease. Furthermore the prevalence and prognosis for some of the 19 medical conditions have changed radically since the origin, and an update of the index is relevant. Other comorbidity indices exist; however the results from most of these are comparable [7, 34, 35].

Survival was assessed as overall and disease-specific mortality. Disease-specific mortality relies on precise and correct data on the cause of death, and particularly in elderly patients where comorbidity is common it can be difficult to achieve 


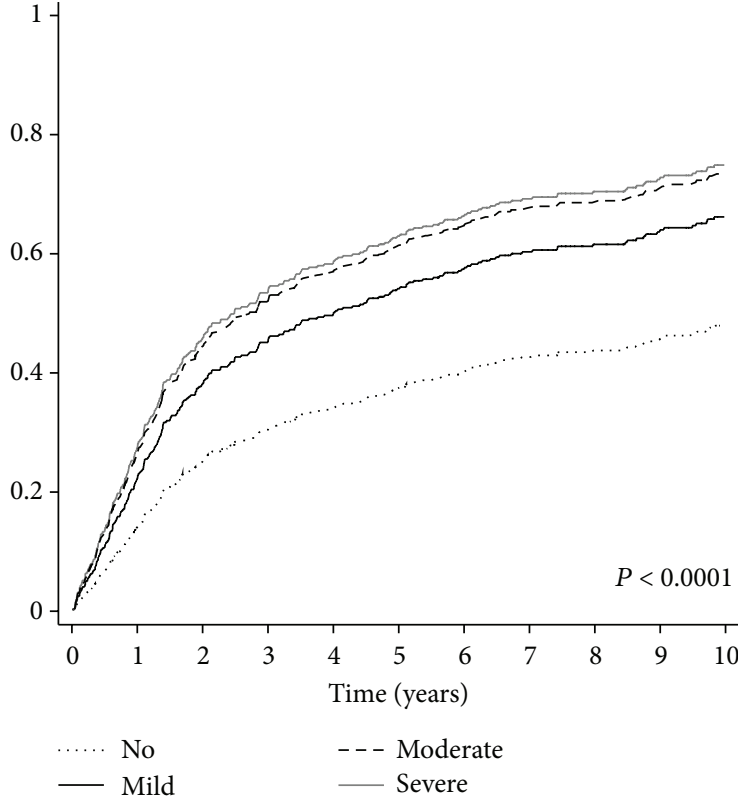

(a)

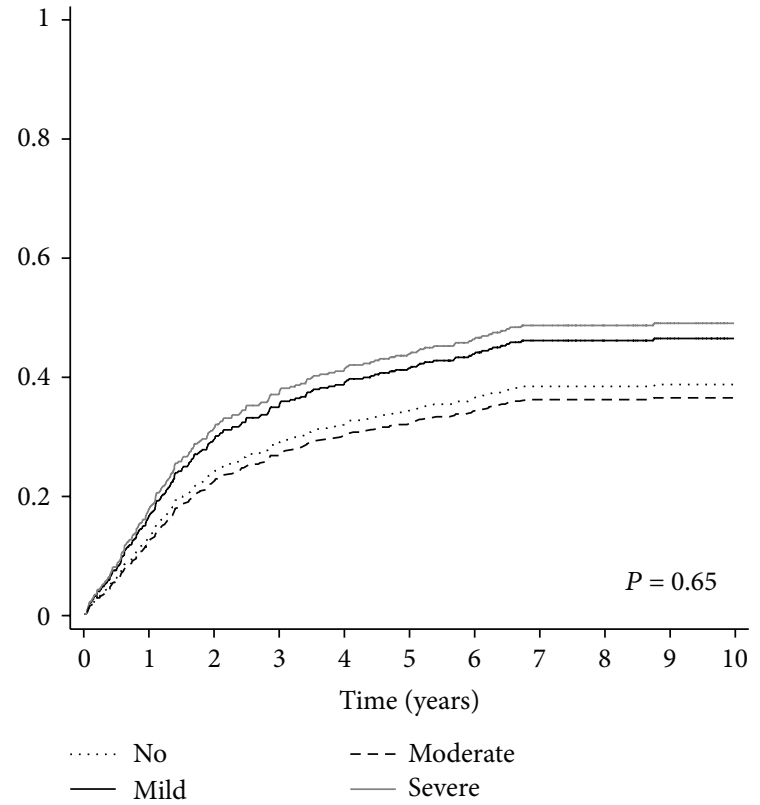

(b)

FIGURE 3: The crude cumulative incidence function of overall (a) and disease-specific mortality (b) by Charlson Comorbidity score.

reliable data. Furthermore in patients with a preceding cancer diagnosis, the risk of stating the cancer as cause of death is increased, causing differential misclassification. Data for the cause of death was retrieved from the ASR in the majority of the cases, where the information is expected to be more precise than the Registry of Cause of Death.

4.2. Prevalence. The prevalence of comorbidity was $19 \%$. The prevalence in our study was low, as expected, since the prevalence of comorbidity increases with increasing age and the median age in our study was only 46 years. The prevalence of comorbidity in bone sarcoma has not previously been investigated, even though a study of 27,506 primary cancer patients (including 413 musculoskeletal tumors) reported comorbidity in $65 \%$ of the overall cases. This study did however include more conditions than the Charlson Comorbidity Index and the musculoskeletal tumor represented only a minor proportion [36]. Comorbidity has been reported to be prevalent in $30-40 \%$ of cases in other cancer types; however, the median age in these types is considerably higher [3-6, 32]. A study of melanoma patients, where $47 \%$ of the patients were younger than 55 years, reported a prevalence of $19 \%$ [37]. We found that the overall prevalence increased over the study period, consistently with the existing literature $[4-6,32]$.

4.3. Survival. The overall and disease-specific mortality rates reported were comparable to the findings of other studies $[38,39]$. The impact of comorbidity was significantly different in patients with Ewing/osteosarcoma histology compared to patients with other subtypes. A tendency towards comorbidity being associated with increased overall and disease-specific mortality was observed in patients with nonEwing/nonosteosarcoma histology. Comorbidity was not associated with neither overall nor disease-specific survival in Ewing/osteosarcoma patients. This might be explained by the low number of patients with comorbidity in this group and thus the low power. Ewing/osteosarcoma patients with severe comorbidity had a surprisingly low overall and diseasespecific mortality. When reviewing these eight patients, three were previously cured from cancer with a good prognosis, and one was primarily diagnosed with an unspecified tumor, later reviewed as Ewing sarcoma. All of these patients are still alive, which might contribute to the low mortality rate.

The impact of comorbidity on survival in bone sarcoma has not, to our knowledge, been investigated. Comorbidity has been found to significantly impact survival in various other cancers [2-6,32].

\section{Conclusion}

The prevalence of comorbidity in adult bone sarcoma patients is low. The level of comorbidity seemingly did not impact the level of treatment in patients with Ewing/osteosarcoma and thus not the disease-specific mortality. Moderate and severe comorbidities were significantly associated with overall survival in patients with non-Ewing/nonosteosarcoma histology, even when adjusting for important prognostic factors. This emphasizes the importance of not only treating the sarcoma but also the comorbidity.

\section{Conflict of Interests}

The authors declare that there is no conflict of interests regarding the publication of this paper. 


\section{Acknowledgments}

This article is a joint first authorship between Katja MarettyNielsen and Ninna Aggerholm-Pedersen. The study was supported by grants from "Frits, Georg \& Marie Cecilie Gluds legat", "Max \& Inge Wørzners mindelegat", the Danish Council for Independent Research | Medical Sciences, and Aarhus University.

\section{References}

[1] K. Maretty-Nielsen, N. Aggerholm-Pedersen, J. Keller, A. Safwat, S. Baerentzen, and A. B. Pedersen, "Population-based aarhus sarcoma registry: validity, completeness of registration, and incidence of bone and soft tissue sarcomas in western Denmark," Journal of Clinical Epidemiology, vol. 5, pp. 45-56, 2013.

[2] M. L. Janssen-Heijnen, S. Houterman, V. E. Lemmens, M. W. Louwman, H. A. Maas, and J. W. Coebergh, "Prognostic impact of increasing age and co-morbidity in cancer patients: a population-based approach," Critical Reviews in Oncology/Hematology, vol. 55, no. 3, pp. 231-240, 2005.

[3] C. R. Boje, S. O. Dalton, T. K. Gronborg et al., "The impact of comorbidity on outcome in 12623 danish head and neck cancer patients: a population based study from the DAHANCA database," Acta Oncologica, vol. 52, no. 2, pp. 285-293, 2013.

[4] L. H. Iversen, M. Nørgaard, J. Jacobsen, S. Laurberg, and H. T. SLrensen, "The impact of comorbidity on survival of danish colorectal cancer patients from 1995 to 2006-a populationbased cohort study," Diseases of the Colon and Rectum, vol. 52, no. 1, pp. 71-78, 2009.

[5] L. Lund, J. Jacobsen, M. Nørgaard et al., "The prognostic impact of comorbidities on renal cancer, 1995 to 2006: a Danish population based study," Journal of Urology, vol. 182, no. 1, pp. 35-40, 2009.

[6] M. S. Tetsche, C. Dethlefsen, L. Pedersen, H. T. Sorensen, and M. Norgaard, "The impact of comorbidity and stage on ovarian cancer mortality: a nationwide Danish cohort study," BMC Cancer, vol. 8, article 31, 2008.

[7] J. F. Piccirillo and I. Costas, "The impact of comorbidity on outcomes," ORL Journal for Oto-Rhino-Laryngology and Its Related Specialties, vol. 66, no. 4, pp. 180-185, 2004.

[8] Statbank Denmark, http://statbank.dk/statbank5a/default.asp? $\mathrm{w}=1280$.

[9] The Danish Civil Registry, http://cpr.dk/cpr/site.aspx?p=194\& ArticleID=4326.

[10] C. B. Pedersen, “The Danish civil registration system," Scandinavian Journal of Public Health, vol. 39, supplement 7, pp. 22-25, 2011.

[11] Danish Health and Medicines Authority, "Cancer pathways, soft tissue and bone sarcoma," http://www.sst.dk/publ/Publ2012/ 06juni/KraeftPkforl/SarkomerKnogleBloeddele3udg.pdf.

[12] Co-operative Cancer Departments, "Treatment of sarcoma and aggressive benign tumors," http://www.ambkir.dk/Faglige\% 20retningslinier/Behandling\%20af\%20sarkomer.pdf.

[13] E. Lynge, J. L. Sandegaard, and M. Rebolj, “The Danish national patient register," Scandinavian Journal of Public Health, vol. 39, supplement 7, pp. 30-33, 2011.

[14] T. N. Nickelsen, "Data validity and coverage in the Danish National Health Registry. A literature review," Ugeskrift for Laeger, vol. 164, no. 1, pp. 33-37, 2001.
[15] J. Mosbech, J. Jørgensen, M. Madsen, K. Rostgaard, K. Thornberg, and T. D. Poulsen, "The national patient registry. Evaluation of data quality," Ugeskrift for Laeger, vol. 157, no. 26, pp. 3741-3745, 1995.

[16] T. F. Andersen, M. Madsen, J. Jørgensen, L. Mellemkjær, and J. H. Olsen, "The Danish National Hospital Register: a valuable source of data for modern health sciences," Danish Medical Bulletin, vol. 46, no. 3, pp. 263-268, 1999.

[17] M. E. Charlson, P. Pompei, K. A. Ales, and C. R. MacKenzie, "A new method of classifying prognostic comorbidity in longitudinal studies: development and validation," Journal of Chronic Diseases, vol. 40, no. 5, pp. 373-383, 1987.

[18] M. S. Tetsche, M. Nørgaard, M. V. Skriver, E. S. Andersen, T. L. Lash, and H. T. Sørensen, "Accuracy of ovarian cancer ICD10 diagnosis in a Danish population-based hospital discharge registry," European Journal of Gynaecological Oncology, vol. 26, no. 3, pp. 266-270, 2005.

[19] K. Helweg-Larsen, "The Danish register of causes of death," Scandinavian Journal of Public Health, vol. 39, supplement 7, pp. 26-29, 2011.

[20] P. K. Andersen, J. P. Klein, and S. Rosthøj, "Generalised linear models for correlated pseudo-observations, with applications to multi-state models," Biometrika, vol. 90, no. 1, pp. 15-27, 2003.

[21] F. Graw, T. A. Gerds, and M. Schumacher, "On pseudo-values for regression analysis in competing risks models," Lifetime Data Analysis, vol. 15, no. 2, pp. 241-255, 2009.

[22] M. Cleves, "Stata54: multiple curves plotted with stcurv command," Stata Technical Bulletin, vol. 9, pp. 7-10, 2000.

[23] E. L. Kaplan and P. Meier, "Nonparametric estimation from incomplete observations," Journal of the American Statistical Association, vol. 53, no. 282, pp. 457-481, 1958.

[24] S. Durrleman and R. Simon, "Flexible regression models with cubic splines," Statistics in Medicine, vol. 8, no. 5, pp. 551-561, 1989.

[25] P. Royston and W. Sauerbrei, "Multivariable modeling with cubic regression splines: a principled approach," Stata Journal, vol. 7, no. 1, pp. 45-70, 2007.

[26] N. Orsini and S. Greenland, "A procedure to tabulate and plot results after flexible modeling of a quantitative covariate," Stata Journal, vol. 11, no. 1, pp. 1-29, 2011.

[27] I. R. White, P. Royston, and A. M. Wood, "Multiple imputation using chained equations: issues and guidance for practice," Statistics in Medicine, vol. 30, no. 4, pp. 377-399, 2011.

[28] J. P. Fine and R. J. Gray, "A proportional hazards model for the subdistribution of a competing risk," Journal of the American Statistical Association, vol. 94, no. 446, pp. 496-509, 1999.

[29] A. D. Oxman and G. H. Guyatt, "A consumer's guide to subgroup analyses," Annals of Internal Medicine, vol. 116, no. 1, pp. 78-84, 1992.

[30] H. T. Sorensen, "Regional administrative health registries as a resource in clinical epidemiology: a study of options, strengths, limitations and data quality provided with examples of use," The International Journal of Risk and Safety in Medicine, vol. 10, no. 1, pp. 1-22, 1997.

[31] S. K. Thygesen, C. F. Christiansen, S. Christensen, T. L. Lash, and H. T. Sørensen, "The predictive value of ICD-10 diagnostic coding used to assess Charlson comorbidity index conditions in the population-based Danish National Registry of Patients," BMC Medical Research Methodology, vol. 11, article 83, 2011.

[32] L. Lund, J. Jacobsen, P. Clark, M. Borre, and M. Nørgaard, "Impact of comorbidity on survival of invasive bladder cancer 
patients, 1996-2007: a Danish population-based cohort study," Urology, vol. 75, no. 2, pp. 393-398, 2010.

[33] T. Nakamura, R. Grimer, C. Gaston et al., "The value of Creactive protein and comorbidity in predicting survival of patients with high grade soft tissue sarcoma," European Journal of Cancer, vol. 49, no. 2, pp. 377-385, 2013.

[34] L. M. Baldwin, C. N. Klabunde, P. Green, W. Barlow, and G. Wright, "In search of the perfect comorbidity measure for use with administrative claims data: does it exist?" Medical Care, vol. 44, no. 8, pp. 745-753, 2006.

[35] V. de Groot, H. Beckerman, G. J. Lankhorst, and L. M. Bouter, "How to measure comorbidity: a critical review of available methods," Journal of Clinical Epidemiology, vol. 56, no. 3, pp. 221-229, 2003.

[36] J. F. Piccirillo, A. Vlahiotis, L. B. Barrett, K. L. Flood, E. L. Spitznagel, and E. W. Steyerberg, "The changing prevalence of comorbidity across the age spectrum," Critical Reviews in Oncology/Hematology, vol. 67, no. 2, pp. 124-132, 2008.

[37] A. F. Grann, T. Froslev, A. B. Olesen, H. Schmidt, and T. L. Lash, "The impact of comorbidity and stage on prognosis of Danish melanoma patients, 1987-2009: a registry-based cohort study," British Journal of Cancer, vol. 109, no. 1, pp. 265-271, 2013.

[38] C. A. Stiller, A. Trama, D. Serraino et al., "Descriptive epidemiology of sarcomas in europe: report from the RARECARE project," European Journal of Cancer, vol. 49, no. 3, pp. 684-695, 2013.

[39] V. Y. Ng, T. J. Scharschmidt, J. L. Mayerson, and J. L. Fisher, "Incidence and survival in sarcoma in the united states: a focus on musculoskeletal lesions," Anticancer Research, vol. 33, no. 6, pp. 2597-2604, 2013. 


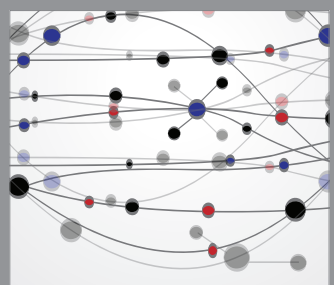

The Scientific World Journal
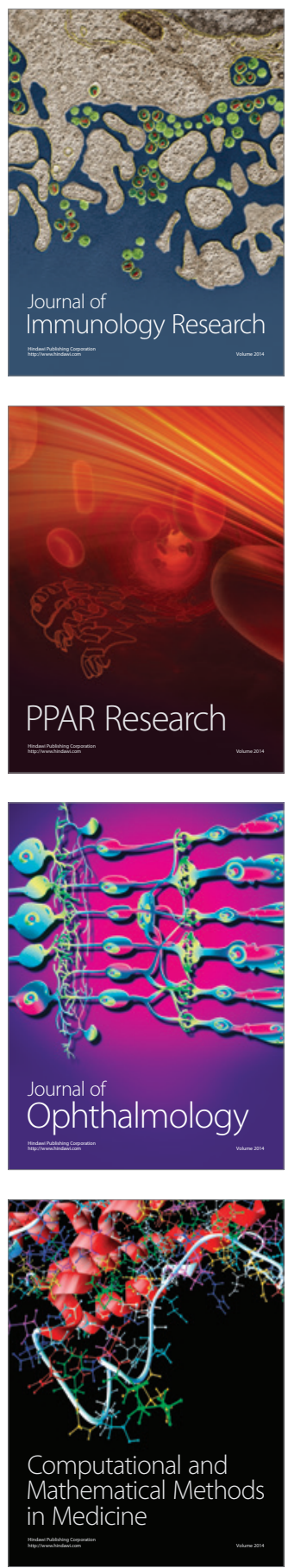

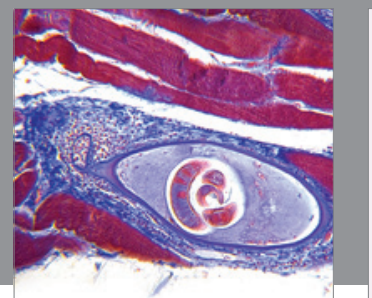

Gastroenterology

Research and Practice
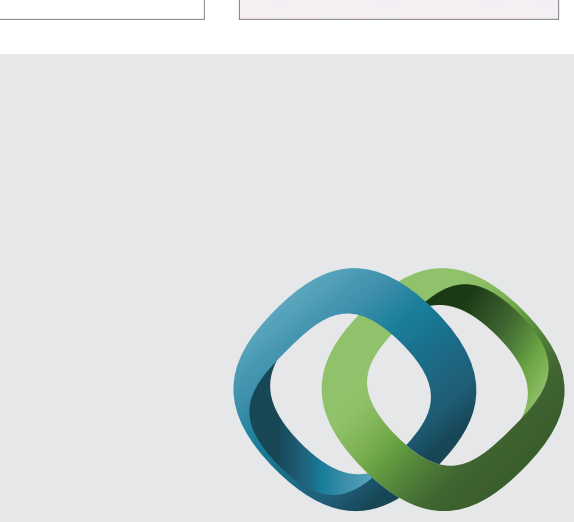

\section{Hindawi}

Submit your manuscripts at

http://www.hindawi.com
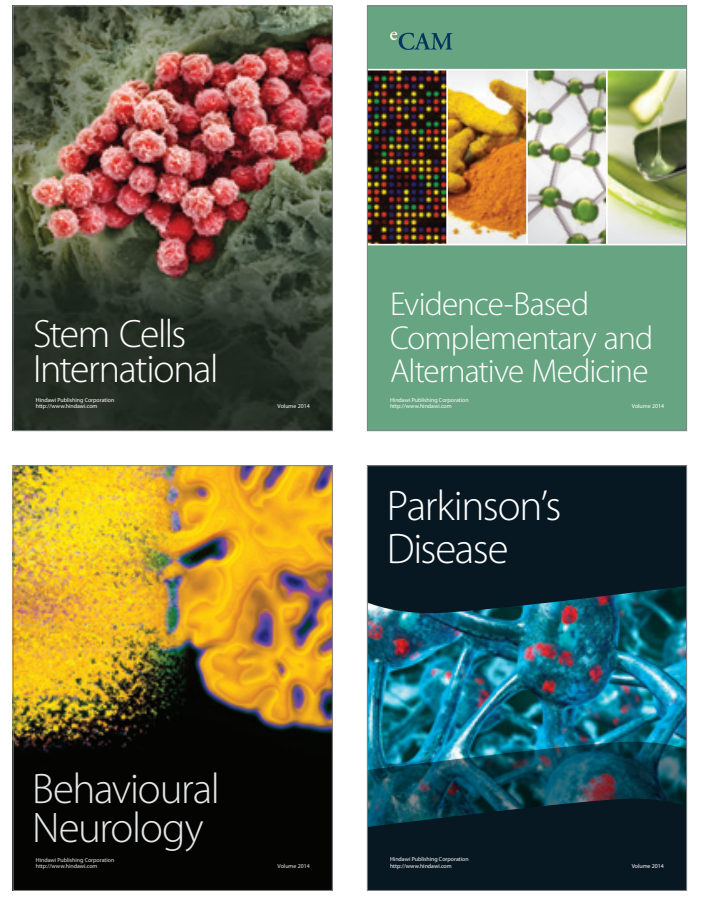
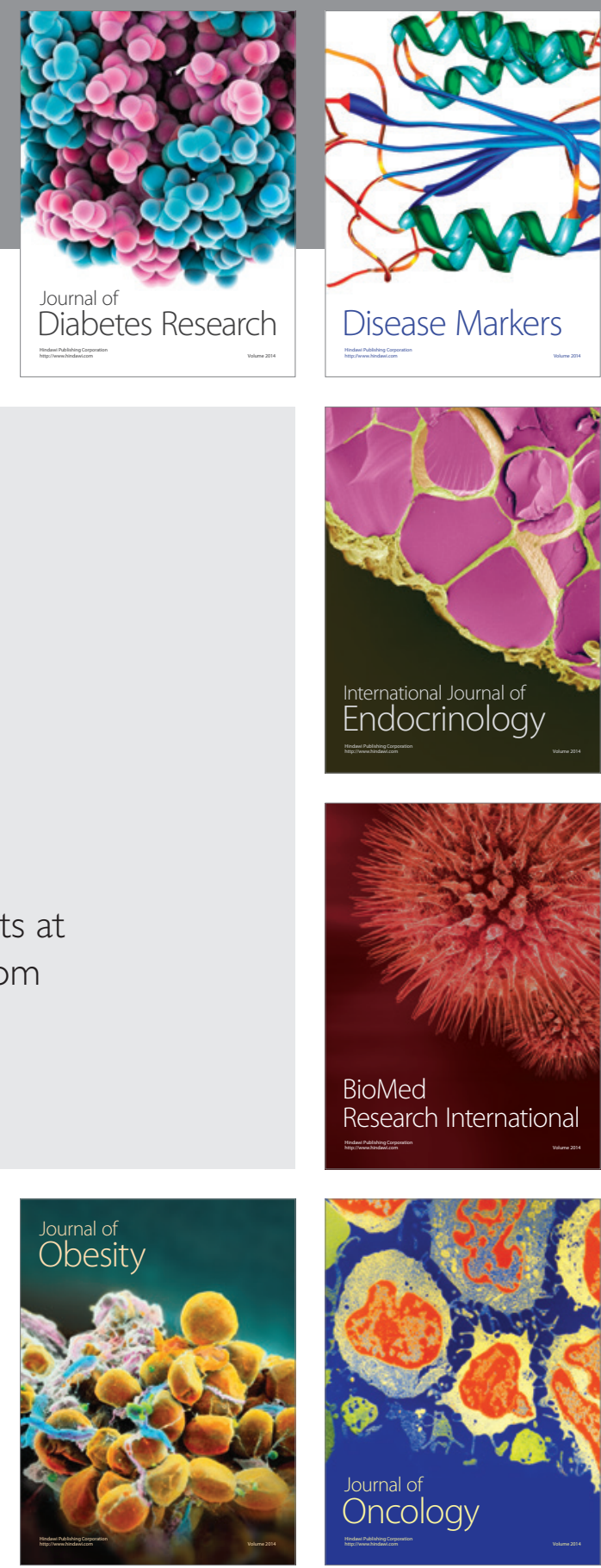

Disease Markers
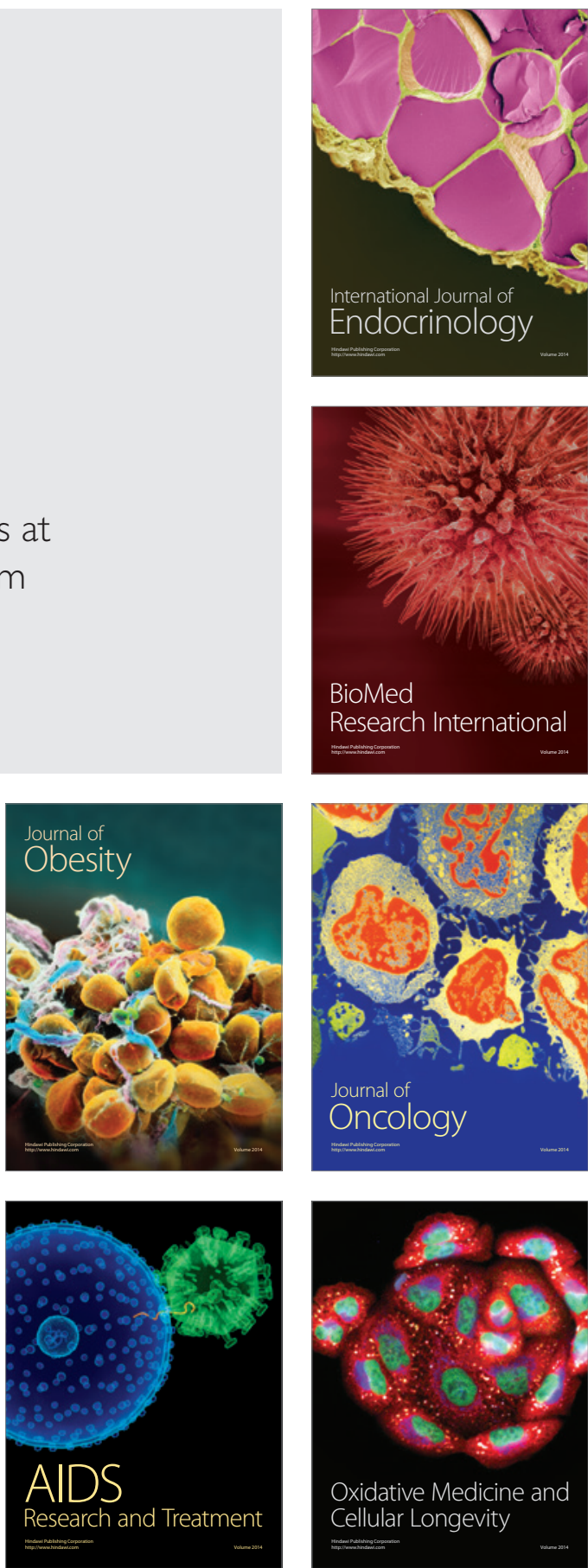